\section{Evitabilidade de óbitos fetais: reflexões sobre a Lista Brasileira de Causas de Mortes Evitáveis por intervenção do Sistema Único de Saúde}

\author{
Avoidability of fetal deaths: reflections on the \\ Brazilian List of Avoidable Causes of Deaths \\ through interventions by the Brazilian Unified \\ National Health System
}

\begin{abstract}
Evitabilidad de óbitos fetales: reflexiones sobre la Lista Brasileña de Causas de Muertes Evitables por intervención del Sistema Único de Salud
\end{abstract}

Sandra Costa Fonseca 1

Pauline Lorena Kale 2

Graça Helena Maia do Canto Teixeira 3

Vania Gloria Silami Lopes 3 doi: 10.1590/0102-311X00265920

\section{Resumo}

Apesar da Lista Brasileira de Causas de Morte Evitáveis (LBE), na sua versão para crianças menores de 5 anos $(L B E<5)$, não contemplar óbitos fetais, alguns estudos a utilizaram sem ou com poucas adaptações. Apresentamos uma proposta de adaptação da LBE para óbitos fetais $(L B E-O F)$ e os resultados de sua aplicação aos óbitos fetais no Estado do Rio de Janeiro, Brasil, em 2018, comparados aos da $L B E<5$. Revisamos as categorias da 10 a revisão da Classificação Internacional de Doenças (CID-10) presentes na $\mathrm{LBE}<5$ e as realocamos nos grupos de evitabilidade, segundo momento do óbito em relação ao parto e as causas condizentes com óbitos fetais. Condições que não esclarecem os determinantes do óbito foram alocadas como causas mal definidas. Óbitos fetais no Estado do Rio de Janeiro - selecionados das bases do Sistema de Informações sobre Mortalidade (SIM) - foram classificados segundo LBE-OF e a $L B E<5$. Ao classificar os 2.585 óbitos fetais do Estado do Rio de Janeiro ocorridos em 2018, observou-se que, segundo a $L B E<5$, predominariam causas "reduzíveis por adequada atenção ao parto" (42,9\%), já pela LBE-OF, aquelas "reduzíveis por adequada atenção à mulher na gestação" foram as mais frequentes $(43,6 \%)$. As causas mal definidas ocuparam a segunda posição segundo a $\operatorname{LBE}-\mathrm{OF}(35,4 \%)$ e a terceira posição segundo $L B E<5$. Cerca de $30 \%$ dos óbitos fetais mudaram de grupos e subgrupos de evitabilidade, mostrando maior coerência com o perfil de atenção obstétrica. Embora identificando um maior percentual de causas mal definidas, a LBE-OF coaduna-se mais com a fisiopatologia dos óbitos fetais. Sua inserção no SIM avançaria no sentido de monitorar e qualificar a investigação de causas de morte fetal.

Morte Fetal; Causas de Morte; Classificação Internacional de Doenças

\author{
Correspondência \\ S. C. Fonseca \\ Instituto de Saúde Coletiva, Universidade Federal Fluminense. \\ Rua Marquês de Paraná 303, 3o andar, Anexo HUAP, Niterói, \\ RJ 24033-900, Brasil. \\ sandracfonseca@yahoo.com.br \\ 1 Instituto de Saúde Coletiva, Universidade Federal Fluminense, \\ Niterói, Brasil. \\ 2 Instituto de Estudos de Saúde Coletiva, Universidade Federal \\ do Rio de Janeiro, Rio de Janeiro, Brasil. \\ 3 Departamento de Patologia, Universidade Federal Fluminense, \\ Niterói, Brasil.
}


Na metade dos anos 2000, os óbitos fetais superaram os óbitos neonatais no Brasil, segundo o Sistema de Informações sobre Mortalidade (SIM) do Departamento de Informática do Sistema Único de Saúde (DATASUS). Em 2005, registravam-se 34.233 óbitos fetais (http://tabnet.datasus.gov.br/cgi/ tabcgi.exe?sim/cnv/fet10uf.def, acessado em 17/Jun/2020) e 34.382 neonatais (http://tabnet.datasus. gov.br/cgi/tabcgi.exe?sim/cnv/inf10uf.def, acessado em 17/Jun/2020); em 2006, este resultado foi invertido, com 33.434 fetais e 32.597 neonatais. Em 2018, esses valores se distanciaram: 30.620 e 25.600 óbitos, respectivamente. A razão óbitos fetais/neonatais, entre 2006 e 2018, aumentou de 1,02 a 1,22 , passando a ser mais que $20 \%$ superior.

Mesmo com números ainda elevados, a taxa de mortalidade fetal (TMF) vem diminuindo, porém em baixa velocidade no país. Em 2012, a taxa nacional era de 10,0/mil nascimentos; as regiões Norte $(10,3 \%$ ) e Nordeste $(12,1 \%$ ) apresentavam os maiores valores, e o Sul o menor $(7,7 \%$ ) 1 . Barros et al. 2 detectaram que a TMF atingiu 9,5/mil nascimentos em 2015, sendo que, de 2000 a 2015, os valores ficaram estacionários e houve grandes diferenças regionais. O comportamento estacionário foi reproduzido entre as causas do capítulo XVI (algumas afecções originadas no período perinatal), da 10a revisão da Classificação Internacional de Doenças (CID-10) 3, que concentra a maioria dos óbitos, exceto para o Nordeste, que mostrou tendência crescente. As malformações congênitas cresceram em todas as regiões do país. Em um estudo ecológico da América Latina, Pingray et al. 4 estimaram a TMF para o Brasil (2015), com magnitude um pouco menor, 8,6\%, e a tendência de redução, entre 2000 e 2015, de 2,3\% anualmente. Fatores associados negativamente ao óbito fetal foram escolaridade materna, produto interno bruto, população urbana e partos hospitalares; já a taxa de fertilidade teve relação positiva com o desfecho.

É importante compreendermos as causas dos óbitos fetais e identificar quais poderiam não ter ocorrido, aplicando classificações de evitabilidade, dimensionando o potencial de prevenção de mortes. Existem várias classificações de causas de óbito que auxiliam a análise dos óbitos fetais 5 , mas nenhuma é considerada ideal. No Brasil, poucos estudos usaram a de Wigglesworth 6 para óbitos perinatais 7,8. E, recentemente, a classificação de evitabilidade - Lista Brasileira de Causas de Morte Evitáveis (LBE) - proposta por Malta et al. 9,10, que lista as causas evitáveis no contexto do Sistema Único de Saúde (SUS) para crianças e adultos, foi aplicada na análise dos óbitos fetais 11,12,13.

A LBE, como outras classificações 14, está alinhada à Classificação Estatística Internacional de Doenças e Problemas Relacionados à Saúde (CID-10) 3, e abrange óbitos nas faixas de 5 a 75 anos e em crianças menores de 5 anos, sem incluir óbitos fetais 9,10. Atualmente, é uma das classificações propostas no módulo de investigação de óbitos infantis e fetais do SIM. A causa básica, definida após a investigação, é utilizada para atribuir ao óbito infantil alguma das categorias de evitabilidade.

Outras características da LBE, além do alinhamento à CID-10, que a tornam particularmente útil, são: (1) diferentes níveis de complexidade, com grupos e subgrupos de causas; (2) regras claras e facilmente aplicáveis; (3) fácil acesso aos dados, por estar disponível no SIM. Uma das limitações, no entanto, é não ser aplicável a óbitos fetais 14 .

Além da LBE não ter sido desenhada para óbitos fetais, algumas inconsistências no preenchimento da causa básica de óbitos fetais já foram apontadas na literatura 7 e em documento do Ministério da Saúde 15. No capítulo sobre a qualidade dos dados dos óbitos fetais, ressalta-se que algumas causas básicas do capítulo XVI (algumas afecções originadas no período perinatal), que se aplicam aos recémnascidos, não são condizentes como causas de óbitos fetais 15. Outras variáveis da declaração de óbito carecem de completude e consistência 15. Variáveis como peso ao nascer e momento do óbito em relação ao parto (antes, durante ou depois), se bem preenchidas, são fundamentais para esclarecer se o óbito foi neonatal ou fetal, direcionar a causa básica e definir o potencial de evitabilidade. A investigação dos óbitos fetais em tempo oportuno e a aplicação sistemática de regras para criticar e supervisionar a qualidade do registro do dado no SIM, contribuem para a maior fidedignidade das informações 15 . No caso de óbito neonatal, o relacionamento com a declaração de nascido vivo pode qualificar os dados 16; para o óbito fetal, a Declaração de Óbito (DO) é, muitas vezes, a única fonte de informação.

Em relação às causas básicas, as mais registradas para óbito fetal no Brasil são "morte fetal de causa não especificada" (21,5\%) e "hipóxia intrauterina não especificada" (15\%), o que denota lacunas no conhecimento 15 e impede a classificação adequada quanto à evitabilidade do óbito.

Outra dificuldade diz respeito ao uso de alguns códigos da CID-10 propostos nos grupos e subgrupos da LBE, que não se aplicariam, do ponto de vista fisiopatológico 17, aos óbitos fetais. 
Grande parte desses óbitos está relacionada a fatores, principalmente maternos, semelhantes aos do óbito neonatal 18 .

Este trabalho teve como principal objetivo propor uma adaptação da LBE para óbitos fetais (LBE-OF). Adicionalmente, avaliamos a mudança na distribuição de causas segundo evitabilidade, comparando a LBE original e a LBE-OF na análise dos óbitos fetais no Estado do Rio de Janeiro, de 2018.

\section{Métodos}

Para a elaboração da LBE-OF, utilizamos como base a LBE atualizada para menores de 5 anos (LBE <5) de 2010 10, que é composta por três grupos de causas de morte: (1) evitáveis; (2) mal definidas e; (3) demais causas ou não claramente evitáveis. O primeiro grupo é subdividido em quatro subgrupos segundo as intervenções do SUS; o segundo subgrupo é subdividido em três categorias: (1.1) reduzíveis por ações de imunoprevenção; (1.2) reduzíveis por adequada atenção à mulher na gestação (1.2.1), parto (1.2.2), feto e ao recém-nascido (1.2.3); (1.3) reduzíveis por ações adequadas de diagnóstico e tratamento e; (1.4) reduzíveis por ações adequadas de promoção à saúde, vinculadas a ações adequadas de atenção à saúde 10. À luz de definições das patologias fetais $17 \mathrm{e}$ das exclusões propostas em trabalhos anteriores 15,19 foram revistas todas as categorias da CID-10 presentes na LBE $<5$, para avaliar a melhor alocação nos grupos de evitabilidade, considerando o momento do óbito (antes do parto ou intraparto) e as causas aplicáveis a óbito fetal.

Algumas premissas gerais nortearam as realocações: condições que não esclarecem as causas determinantes do óbito foram alocadas no grupo de causas mal definidas. Aqui se incluem alguns códigos cujo quarto dígito é o numeral 9; em geral referente a causas não especificadas. Condições exclusivamente relacionadas ao cuidado ao recém-nascido foram consideradas inconsistentes e alocadas no mesmo grupo de mal definidas, como um item diferenciado.

Dois códigos agrupados como redutíveis por atenção ao feto e ao recém-nascido relacionados a diabetes mellitus materna foram redistribuídos para o grupo de redutíveis por atenção à gestação, considerando que só poderia haver prevenção do óbito fetal durante a gravidez ${ }^{19}$. Outros códigos foram considerados como não aplicáveis ao feto, por se relacionarem a quadros mórbidos presentes apenas em recém-nascidos. Esses códigos, quando presentes na declaração de óbito, foram incluídos no grupo de causas mal definidas.

Com base nos dados de mortalidade fetal do SIM, disponibilizados no sítio do DATASUS (http:// www2.datasus.gov.br/DATASUS/index.php?area $=0205 \& \mathrm{id}=6937$, acessado em 02/Jul/2020), selecionamos óbitos fetais com 22 semanas de gestação ou mais e aqueles com idade gestacional ignorada, no Estado do Rio de Janeiro, 2018, e aplicamos a LBE-OF e a LBE < 5, para fins de comparação. Foram calculadas a mortalidade proporcional por grupo e subgrupo da LBE-OF e da LBE $<5$ e a proporção de óbitos fetais que apresentaram mudança de classificação de evitabilidade por grupos e subgrupos, quando aplicada a LBE-OF.

Foram avaliados também a qualidade dos registros por meio da descrição absoluta e o porcentual das inconsistências em relação ao momento do óbito fetal (antes, durante, depois do parto e ignorado/ em branco), do responsável pelo preenchimento (serviços de saúde de ocorrência do óbito ou serviço de verificação do óbito/Instituto Médico Legal - SVO/IML) e a realização de investigação epidemiológica (sim/não).

Para avaliar a significância estatística das mudanças entre as duas classificações, nos óbitos fetais no Estado do Rio de Janeiro, aplicamos o teste McNemar para amostras pareadas (antes e depois, considerando antes grupos e subgrupos da $\mathrm{LBE}<5$ e depois, LBE-OF).

O estudo é parte integrante da pesquisa Estudo Sobre Indicadores de Saúde da Mulher e da Criança nas Regiões de Saúde do Estado do Rio de Janeiro, aprovada pelo Comitê de Ética em Pesquisa da Faculdade de Medicina, Universidade Federal Fluminense (parecer no 4.091.556). 


\section{Resultados}

\section{Adaptação da LBE para óbitos fetais (LBE-OF)}

Do item 1.1 da LBE < 5, "reduzíveis por ações de imunoprevenção", mantivemos as patologias relacionadas à transmissão vertical, que podem comprometer o feto e que dispõem de vacina eficaz: rubéola congênita (P35.0); hepatite B (B16). As demais, que dependem da vacinação da criança após o nascimento, foram excluídas, consideradas inconsistentes com o óbito fetal (Quadro 1).

As condições do grupo "reduzíveis por adequada atenção à mulher na gestação, parto, feto e ao recém-nascido" foram analisadas quanto à presença de comprometimento fetal antes e durante o parto e a possibilidade de prevenção; as condições dependentes de cuidados pós-natais foram consideradas inconsistentes com o óbito fetal (Quadro 1).

Ao subgrupo "reduzíveis por adequada atenção à mulher na gestação", além das infecções constantes da $\mathrm{LBE}<5$, acrescemos tuberculose congênita (P37.0); toxoplasmose congênita (P37.1); malária congênita (P37.3 e P37.4) (originalmente 1.4); e infecção intra-amniótica do feto (P39.2); do subgrupo 1.2.2 trouxemos transtornos relacionados com pós-maturidade (P08.2); do subgrupo 1.2.3 trouxemos síndrome do filho de mãe com diabetes gestacional (P70.0); síndrome do filho de mãe diabética

\section{Quadro 1}

Lista Brasileira de Causas de Mortes Evitáveis para óbitos fetais (LBE-OF).

\section{Causas evitáveis}

1.1. Reduzíveis por ações de imunoprevenção: rubéola congênita (P35.0); hepatite viral congênita (P35.3).

1.2. Reduzíveis por adequada atenção à mulher na gestação, parto, feto e ao recém-nascido

1.2.1. Reduzíveis por adequada atenção à mulher na gestação

- Sífilis congênita (A50, A50.0, A50.1, A50.2); doenças pelo vírus da imunodeficiência humana (B20 a B24);

- Feto e recém-nascidos afetados por afecções maternas, não obrigatoriamente relacionadas com a gravidez (P00), feto e recém-nascidos afetados por complicações da placenta e das membranas (P02.2, P02.3, P02.7, P02.8, P02.9); feto ou recém-nascido afetado por influências nocivas via placenta (P04); complicações maternas da gravidez que afetam o feto ou o recém-nascido (P01); crescimento fetal retardado e desnutrição fetal (P05); transtornos relacionados com a gestação de curta duração e baixo peso ao nascer, não classificados em outra parte (P07); perda sanguínea fetal (P50); hemorragia intracraniana não traumática do feto e do recém-nascido (P52); isoimunizaçao Rh e ABO do feto ou do recém-nascido (P55.0, P55.1); doenças hemolíticas do feto ou do recém-nascido devidas à isoimunizaçao (P55.8 a P57.9).

Incluídos:

- Transtornos relacionados com pós-maturidade (P08.2) (originalmente subgrupo 1.2.2);

- Tuberculose congênita (P37.0); toxoplasmose congênita (P37.1); malária congênita (P37.3 e P37.4); infecção intra-amniótica do feto originalmente do subgrupo (P39.2); síndrome do filho de mãe com diabetes gestacional (P70.0); síndrome do filho de mãe diabética (P70.1); interrupção da gravidez (P96.4); e complicações procedimentos intrauterinos (P96.5) (originalmente subgrupo 1.2.3).

1.2.2. Reduzíveis por adequada atenção à mulher no parto

Placenta prévia e descolamento prematuro da placenta (P02.0 a P02.1); feto e recém-nascido afetados por afecções do cordão umbilical (P02.4 a P02.6); outras complicações do trabalho de parto ou do parto que afetam o feto e o recém-nascido (P03); transtornos relacionados com gestação prolongada e peso elevado ao nascer (P08; exceto P08.2, realocado para o subgrupo 1.2.1); traumatismo de parto (P10 a P15); hipóxia intrauterina antes do início do trabalho de parto (P20.0, exceto P20.9 realocado para o grupo 2 mal definidas) e durante o trabalho de parto (P20.1).

1.2.3. Reduzíveis por adequada atenção ao feto e ao recém-nascido

A maioria dos códigos deste subgrupo não se aplica aos óbitos fetais, exceto P35.1, P35.2, P35.8, P35.9, realocados para o grupo 3; P37.0, P37.1, P37.3, P37.4, P39.2, P70.0, P70.1, P83.3, P96.4 e P96.5, realocados para 1.2.1.

1.3. Reduzíveis por ações adequadas de diagnóstico e tratamento - não se aplicam.

1.4. Reduzíveis por ações adequadas de promoção à saúde e atenção à saúde - não se aplicam. 


\section{Causas de mortes mal definidas ou inconsistentes com óbito fetal}

Mal definidas - morte fetal de causa não especificada (P95); outras afecções originadas no período perinatal (P96.8); afecções originadas no período perinatal, não especificadas (P96.9).

Sintomas, sinais e achados anormais de exames clínicos e de laboratório não classificados em outra parte (R00 a R99).

Incluídos:

- Hipóxia intrauterina, não especificada (P20.9) (originalmente do subgrupo 1.2.2.); hidropisia fetal não devida à doença hemolítica (P83.2); e outros edemas e os não especificados do feto (P83.3) (originalmente do subgrupo 1.2.1.).

Inconsistentes:

- Tuberculose do sistema nervoso (A17); tuberculose miliar (A19); tétano neonatal (A33); outros tipos de tétano (A35); difteria (A36); coqueluche (A37); poliomielite aguda (A80); sarampo (B05); rubéola (B06); hepatite B (B16); caxumba (B26.0); e meningite por haemophilus (G00.0) (originalmente do grupo 1).

- Asfixia ao nascer (P21) e P24 (originalmente do subgrupo 1.2.2.).

- Transtornos respiratórios específicos do período perinatal (P22.1, P22.8, P22.9, P23, P25, P27, P28, originalmente do subgrupo 1.2.3).

- P26 originalmente do subgrupo 1.2.1.).

- Infecções especificas do período perinatal (P36 a P39.9, exceto: P37.0, 37.1, 37.3, 37.4 e 39.2 que foram realocados para o subgrupo 1.2.1); hemorragia neonatal (P51,P53, P54, e outras icterícias perinatais (P58, P59); transtornos endócrinos e metabólicos transitórios específicos e do recém-nascido (P70 a P74; exceto $P 70.0$ e 70.1, realocados para 1.2.1.); transtornos hematológicos do recém-nascido (P60, P61); transtornos do aparelho digestivo do recém-nascido (P75 a P78); afecções que comprometem o tegumento e a regulação térmica do recém-nascido (P80 a P83; exceto P83.2 e 83.3, realocados para grupo 2.); outros transtornos originados no período perinatal - convulsões do recém-nascido (P90); outros distúrbios da função cerebral neonatal (P91); problemas de alimentação (P92); reações a intoxicações (P93); transtornos do tônus do recémnascido (P94); outras afecções do período perinatal (P96.0 a P96.3) ( originalmente do subgrupo 1.2.3.).

3. Demais causas (não claramente evitáveis)

Malformações graves (principais: Q00; Q20-Q24; Q33.3; Q33.6; Q60-Q61; Q79.0; Q79.1; Q79.3; Q89; Q91).

Incluídos:

- Infecção congênita por citomegalovírus (P35.1); infecção congênita por herpes (P35.2); varicela congênita (P35.8) *; e doenças virais congênitas e não especificadas (P35.9) (originalmente do subgrupo 1.2.3.).

* A vacina tetraviral com o componente da varicela entrou no calendário do Ministério da Saúde em 2013, espera-se diminuição da varicela em mulheres em idade fértil, podendo o agravo ser reavaliado como causa evitável de perda fetal.

(P70.1); interrupção da gravidez (P96.4); e complicações de procedimentos intrauterinos (P96.5). Excluímos síndrome da angústia respiratória do recém-nascido (P22), hemorragia pulmonar (P26), enterocolite necrotizante do feto e do recém-nascido, por se aplicarem exclusivamente ao recém-nascido (P77) (Quadro 1). As demais condições foram mantidas.

Para o subgrupo "reduzíveis por adequada atenção à mulher no parto", a maior alteração diz respeito à hipóxia intrauterina não especificada (P20.9), que foi excluída e realocada para causas mal definidas (Quadro 1). As demais condições foram mantidas.

Para as condições do subgrupo "reduzíveis por adequada atenção ao feto e ao recém-nascido", vários códigos foram realocados. Dois códigos relacionados a diabetes mellitus materna, síndrome do filho de mãe com diabetes gestacional (P70.0) e síndrome do filho de mãe diabética (P70.1) foram redistribuídos para o grupo 1.2.1. Outros códigos foram considerados como não aplicáveis ao feto, por se relacionarem a quadros mórbidos presentes apenas em recém-nascidos. Todas as afecções pulmonares que dependem de tempo de vida para se manifestar, como as pneumonias congênitas (P23), enfisema intersticial e correlatos (P25), doença respiratória crônica (P27), outras afecções respiratórias (P28), como atelectasia e apneia do recém-nascido, foram excluídas. Da mesma forma, excluímos a maioria das infecções específicas perinatais, manifestas no recém-nascido (exceções já explicitadas anteriormente), a hemorragia neonatal (P50-P54), as icterícias perinatais (P58, P59), os transtornos digestivos do recém-nascido (P75-P78), os transtornos do tegumento (exceto P83.0 e P83.2) e regulação térmica (P80-83), e os transtornos metabólicos do recém-nascido (exceto P70.0 e P70.1). Esses códigos, quando presentes na declaração de óbito, foram considerados inconsistentes e incluídos no grupo de causas mal definidas (Quadro 1). 
Outras condições (grupos 1.3 e 1.4 da LBE < 5), que claramente são relacionadas a intervenções em crianças de forma individual e/ou populações, foram avaliadas e, aquelas que poderiam comprometer o feto, foram realocadas para o subgrupo item 1.2.1 porque seriam redutíveis por cuidados à gestante. As demais foram consideradas inconsistentes com o óbito fetal (Quadro 1).

\section{Causas evitáveis de morte fetal no Estado do Rio de Janeiro}

Ocorreram 2.585 óbitos fetais de mães residentes no Estado do Rio de Janeiro em 2018, dos quais, 2.336 (90,4\%) registrados como antes do parto, 130 (5\%) durante o parto, nenhum depois do parto, e em 199 (4,6\%), o dado estava ausente. O responsável pelo preenchimento foi majoritariamente o médico lotado nos hospitais, apenas 50 óbitos tinham como declarante o SVO ou o IML. Cerca de $80 \%$ dos óbitos foram investigados, $25 \%$ tinham peso ao nascer igual ou maior que 2.500g; mais da metade ultrapassou o peso de $1.500 \mathrm{~g}$ (55\%).

$\mathrm{Na}$ Tabela 1 são apresentadas as distribuições dos óbitos fetais segundo causas evitáveis, de acordo com a LBE < 5 anos e LBE-OF. Quanto às causas evitáveis, predominaram as "reduzíveis por adequada atenção à mulher no parto", quando aplicada a LBE < 5 (42,9\%), e "reduzíveis por adequada atenção à mulher na gestação", quando aplicada a LBE-OF (43,6\%). As causas mal definidas ocuparam a segunda posição de maior frequência segundo a LBE-OF e a terceira segundo a LBE < 5. Não ocorreu óbito fetal por causas reduzíveis por imunoprevenção nem por ações de promoção à saúde; apenas um óbito (Q90.9) do grupo de reduzíveis por ações de diagnóstico e tratamento da $\mathrm{LBE}<5$. A proporção de óbitos por demais causas praticamente não alterou quando utilizadas a $\mathrm{LBE}<5(5,6 \%)$ e a $\mathrm{LBE}-\mathrm{OF}(5,8 \%)$.

Houve mudança de grupos e subgrupos de evitabilidade de $31 \%$ dos óbitos fetais quando aplicadas a $\mathrm{LBE}<5$ e a LBE-OF (Figura 1$)$. O maior percentual de mudança $(28,7 \%)$ ocorreu com a migração de causas específicas do subgrupo "reduzíveis por adequada atenção à mulher no parto" $(\mathrm{LBE}<5)$ para "mal definidas ou inconsistências com óbito fetal” (LBE-OF).

Tabela 1

Distribuição dos óbitos fetais ( $n=2.585$ ) no Estado do Rio de Janeiro, Brasil, em 2018, segundo a Lista Brasileira de Causas de Mortes Evitáveis para menores de 5 anos (LBE < 5) e para óbitos fetais (LBE-OF).

\begin{tabular}{|c|c|c|c|c|}
\hline \multirow[t]{2}{*}{ Classificação de evitabilidade de causas de morte } & \multicolumn{2}{|c|}{ LBE $<5$} & \multicolumn{2}{|c|}{ LBE-OF } \\
\hline & $\mathbf{n}$ & $\%$ & $\mathbf{n}$ & $\%$ \\
\hline \multicolumn{5}{|l|}{ 1. Causas evitáveis } \\
\hline 1.1. Reduzíveis por ações de imunoprevenção & 0 & 0,00 & 0 & 0,00 \\
\hline \multicolumn{5}{|c|}{ 1.2. Reduzíveis por adequada atenção à mulher na gestação, no parto e ao recém-nascido } \\
\hline 1.2.1. Reduzíveis por adequada atenção à mulher na gestação & 1.076 & 41,63 & 1.127 & 43,6 \\
\hline 1.2.2. Reduzíveis por adequada atenção à mulher no parto & 1.108 & 42,86 & 392 & 15,16 \\
\hline 1.2.3. Reduzíveis por adequada atenção ao recém-nascido & 81 & 3,13 & 0 & 0,00 \\
\hline 1.3. Reduzíveis por ações adequadas de diagnóstico e tratamento & 1 & 0,04 & NA & NA \\
\hline 1.4. Reduzíveis por ações adequadas de promoção à saúde e atenção à saúde & 0 & 0,00 & NA & NA \\
\hline 2. Causas de morte mal definidas & 174 & 6,73 & 917 & 35,48 \\
\hline 3. Demais causas (não claramente evitáveis) & 145 & 5,61 & 149 & 5,76 \\
\hline
\end{tabular}

NA: não se aplica. 
Figura 1

Mudanças das causas específicas e os respectivos códigos da 10ª revisão da Classificação Internacional de Doenças (CID-10) por grupos e subgrupos de evitabilidade da Lista Brasileira de Causas de Mortes Evitáveis para menores de 5 anos (LBE < 5) e para óbitos fetais (LBE-OF). Estado do Rio de Janeiro, Brasil, 2018.

\section{LBE $<5$}

\subsection{3) Reduzíveis por adequada atenção ao recém-nascido:}

P3.71 - toxoplasmose congênita $(n=1)$, P37.9 - doença infecciosa ou parasitária congênita não especificada $(n=1)$, P39.2 - infecção intra-amniótica do feto não classificada em outra parte $(n=1), P 70.0$ - síndrome do filho de mãe com diabetes gestacional $(n=31), P 70.1$ síndrome do filho de mãe diabética $(n=11)$, P96.4 - interrupção de gravidez afetando o feto e o recém-nascido $(n=6)$ e P96.5 - complicações de procedimentos intrauterinos não classificados em outra parte $(n=2)$.

\subsection{2) Reduzíveis por adequada atenção à mulher no parto:}

P20.9 - hipóxia intrauterina não especificada $(n=716)$.

\subsection{3) Reduzíveis por adequada atenção ao recém-nascido:}

P83.2 - hidropsia fetal não-devida à doença hemolítica $(n=23)$, P96.8 - outras afecções especificadas originadas no período perinatal $(n=1)$, P70.9 - transtorno transitório não especificado do metabolismo dos carboidratos do feto e do recém-nascido $(n=1)$ e P83.5 - hidrocele congênita $(n=1)$.

\section{3) Reduzíveis por ações adequadas de diagnóstico e tratamento:}

Q90.9 - síndrome de Down não especificada $(n=1)$.

\subsection{1) Reduzíveis por adequada atenção à mulher na gestação:}

P56.0 - hidropisia fetal devida à isoimunização $(n=1)$ e P56.9 - hidropisia fetal devida a outras doenças hemolíticas e às não especificadas $(n=1)$.

\subsection{3) Reduzíveis por adequada atenção ao recém-nascido:}

P35.1 - infecção congênita por citomegalovírus $(n=1)$ e P35.8 - outras doenças virais congênitas $(n=1)$.

\section{LBE-OF}

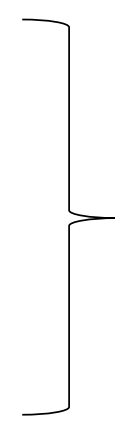

1.2.1) Reduzíveis por adequada atenção à mulher na gestação

$$
n=53(2,1 \%) \text { * }
$$

\section{Causas de morte mal definidas}

$n=743(28,7 \%)$ *

3. Demais causas (não claramente evitáveis)

$n=4(0,2 \%)$ *

Total de mudanças

$\mathrm{n}=\mathbf{8 0 0}(\mathbf{3 1 \%})$

* Teste de McNemar $(p<0,0001)$. 


\section{Discussão}

O estudo mostrou a factibilidade de aplicação da LBE aos óbitos fetais e as mudanças decorrentes da distribuição de causas evitáveis, destacando-se o maior percentual atribuível a causas maternas.

Parte da aplicabilidade é explicada pela facilidade inerente à LBE em classificar óbitos de acordo com a CID-10, dispensando informações por vezes ausentes ou não consistentes na declaração de óbito 9,10. Sempre deve ser almejada a investigação completa das condições do óbito, do status sociodemográfico materno e de detalhes da assistência prestada. A identificação de desigualdades no acesso aos cuidados de saúde depende do adequado preenchimento de variáveis como idade, cor da pele e escolaridade 7. No entanto, mesmo na ausência dessas variáveis, as categorias da LBE-OF poderão identificar dimensões deficientes da assistência e contribuir para direcionar as políticas de saúde materna e infantil.

A ampliação das causas relacionadas a afecções maternas obtidas com a LBE-OF corrobora a maioria dos estudos na área perinatal 20,21,22,23,24. A Organização Mundial da Saúde (OMS) 25, em 2016, reafirmou a necessidade de valorizar as causas maternas na DO e/ou na investigação de óbitos perinatais, adicionando um campo para a condição materna mais relevante para o óbito, ou, pelo menos, o preenchimento da causa básica com esta condição.

A LBE-OF resultou em um maior percentual de causas mal definidas no estado analisado - cerca de 35\% -, o que corroborou a análise nacional, na qual a causa hipóxia intrauterina não especificada (P20.9) ocorreu em 15\% dos óbitos fetais em 2017, considerada mal definida 15. Uma revisão sistemática de óbitos fetais em países de baixa e média rendas encontrou percentuais entre 3,8\% e 57,4\% de casos não classificáveis 20 . No grupo de mal definidas, cabe uma separação entre aquelas oriundas de causas não especificadas e outras, que denominamos inconsistentes 15 , provenientes da atribuição de causas neonatais aos óbitos fetais. Optamos por mantê-las juntas, preservando a estrutura da LBE original. Mas essas categorias devem ser sempre exploradas, para avaliar qual o peso de cada tipo de má classificação.

Alocar o código P20.9 no grupo de mal definidas foi a principal diferença entre os nossos resultados e os encontrados na LBE $<5$ 11,12,13. Em Belo Horizonte, de 2008 a 2010, a primeira causa foi o grupo de reduzíveis por atenção ao parto, considerando os códigos P20 e 21, sem detalhamento 11. Em Pernambuco, de 2009 a 2011, o principal subgrupo (34,2\%) foi de evitáveis por atenção ao parto, destacando-se cerca de 1.000 óbitos fetais, com os códigos P20-P21, agrupados indiscriminadamente 12. Em Recife, de 2010 a 2014, predominaram as causas evitáveis por atenção à gestante, seguidas das reduzíveis por atenção ao parto, nas quais foram incluídas hipóxia intrauterina e asfixia ao nascer, sem especificar os códigos 13 .

Em nosso estudo, ao se aplicar a $\mathrm{LBE}<5$, da mesma forma que os três estudos citados, o código P20.9, alocado nas causas redutíveis por adequada atenção ao parto, destacou-se na primeira posição, respondendo por $67 \%$ das causas neste subgrupo de evitabilidade, ocupando o posto de mais frequente na análise do Estado do Rio de Janeiro. Classificar o código P20.9 nesse grupo de evitabilidade atribui equivocadamente esses óbitos a falhas no momento do parto, desviando em parte o foco dos atrasos na assistência obstétrica 26. Muitos desses óbitos correspondem a mulheres que chegam às maternidades com o feto morto 20,27. Recuperar informações sobre a trajetória reprodutiva dessa mulher desde o pré-natal, passando pela peregrinação por outras unidades de saúde, até o cuidado recebido na admissão hospitalar 26 deve ser o alvo dos comitês de investigação do óbito fetal. Apenas 130 óbitos foram descritos como ocorridos durante o parto. Essa incongruência corrobora a inadequação da classificação de "reduzíveis por adequada atenção à mulher no parto", em elevado percentual dos óbitos, segundo a LBE < 5. A LBE-OF não modifica esse dado, mas classifica essas causas como mal definidas. Entre os óbitos fetais classificados segundo a LBE-OF, não ocorreu nenhuma causa considerada inconsistente com o óbito fetal (dados não apresentados), minimizando a possibilidade de erro de classificação entre nascidos vivos e óbitos fetais na base de dados.

No Estado do Rio de Janeiro, ao aplicar a LBE-OF, o grupo de mal definidas passou a ocupar a segunda posição, e as redutíveis por atenção à gestante o primeiro lugar. Mesmo quando aplicamos a $\mathrm{LBE}<5$, identificou-se frequência elevada de causas redutíveis por adequada atenção à gestante. Apesar da cobertura ampliada no país 28,29 , persiste a irregularidade da qualidade do pré-natal oferecido no Estado do Rio de Janeiro, tanto quantitativa como qualitativa 30,31,32, o que favorece desfechos 
graves maternos e fetais 18,20. Com a LBE-OF, a proporção para esse grupo de reduzíveis por atenção à gestante foi ainda maior, graças à alocação mais adequada de alguns códigos da CID-10.

Na LBE-OF, as causas de morte evitáveis por imunoprevenção ficaram restritas à rubéola congênita (P35.0) e hepatite viral congênita (P35.3). Varicela congênita (P35.8) foi alocada para o grupo de Demais causas ou não claramente evitáveis porque, apesar da disponibilidade da vacina no SUS, a introdução aconteceu a partir de 2013. Ainda há coortes de gestantes suscetíveis à doença, mas, futuramente, varicela congênita poderá ser realocada para o subgrupo de imunoprevenção. Os subgrupos de causas evitáveis por ações adequadas de diagnóstico e tratamento (subgrupo 1.3) e de promoção à saúde, e atenção à saúde (subgrupo 1.4) da LBE em $<5$ foram considerados inaplicáveis aos óbitos fetais. Todos os demais grupos e subgrupos apresentaram migração de códigos na adaptação para óbitos fetais.

Quanto aos resultados do Estado do Rio de Janeiro, identificou-se, além da necessidade de mais investimentos no pré-natal, os déficits no preenchimento da DO e no processo de investigação dos óbitos fetais. Os resultados de outros locais podem ser diferentes, de acordo com o cenário de atenção perinatal e a qualidade dos dados. Um estudo recente, comparando maternidades das cidades de São Paulo e do Rio de Janeiro, com uma proposta semelhante de LBE adaptada, identificou mais problemas de atenção ao parto na primeira, e mais deficiências do pré-natal no Rio de Janeiro 16.

A mortalidade fetal vem suplantando a neonatal no Brasil e esta transição requer o fortalecimento de estratégias voltadas ao óbito fetal 33 . Conhecer as causas da natimortalidade é crucial para o enfrentamento de desfecho tão grave e frequente.

A LBE é a classificação mais usada no país 11,12,13,21,22,23,24 e tem muitas características que a habilitam como um "sistema global de classificação" 14 . Contextualizada ao perfil de atendimento do SUS, pode ser usada também como parâmetro em avaliações de intervenções de saúde 24 .

A adaptação para óbitos fetais avança no sentido de identificar, com maior consistência, causas de mortes fetais preveníveis por tecnologia disponível no SUS e apontar falhas da assistência prestada. Para isso, é imprescindível a informação de qualidade na declaração de óbito, destacando o correto preenchimento das causas de mortes e demais variáveis relacionadas. Uma lista diferenciada trará maior visibilidade dos óbitos fetais e ampliará a compreensão das causas de morte fetal, como transtornos metabólicos e infecciosos, dependentes da atenção à gestante.

Uma limitação do trabalho foi não incluir a análise por peso ao nascer e a duração da gestação. $O$ percentual de ignorados dessas variáveis foi baixo e um quarto dos óbitos fetais tinha peso adequado, sugerindo ainda mais o potencial de evitabilidade. Quanto à duração da gestação, não identificamos estudos sobre o SIM, mas, no Sistema de Informações sobre Nascidos Vivos (SINASC), tem sido uma variável com baixa consistência 34 . Reforçamos a importância da avaliação da evitabilidade segundo essas variáveis e da qualidade desses dados 35 .

Para futuras versões da DO, deve-se ampliar o bloco IV, com informações maternas (cor da pele) e detalhamento do campo 34, principalmente para o óbito fetal anteparto, detalhando se ocorreu antes do trabalho de parto ou antes da internação hospitalar. Muitas gestantes não são atendidas na primeira maternidade, tendo de procurar por duas ou mais até conseguir internação, portanto, um óbito anteparto pode ter ocorrido intraparto e estar mais relacionado à peregrinação do que ao atendimento pré-natal. Além disso, a adoção, neste bloco, de um campo específico para as causas maternas, como proposto pela OMS, pode ser de grande valia para a definição adequada da causa básica do óbito e para os comitês de investigação do óbito fetal e infantil 25,36.

Os comitês de investigação de óbito fetal e infantil devem ser valorizados e receber mais recursos humanos. Segundo a literatura, o esforço dos comitês tem se direcionado mais à análise de óbitos infantis, em detrimento dos fetais, não só no Brasil, mas em outros países desenvolvidos ou em desenvolvimento ${ }^{37}$. A "invisibilidade" dos óbitos fetais, comparada à de mortes maternas e infantis, precisa ainda ser enfrentada $35,38,39$. A classificação da evitabilidade é uma etapa importante da vigilância dos óbitos, sejam fetais ou não, e depende da qualidade dos dados e da instrumentalização dos comitês e secretarias para ampliar o alcance da investigação. A vigilância do óbito fetal, obrigatória no Brasil, é crucial na identificação das falhas assistenciais e na redução da mortalidade 35,38,39. Por último, recomenda-se a inclusão da LBE-OF no SIM, de forma semelhante ao que ocorre para os óbitos não fetais. 


\section{Colaboradores}

S. C. Fonseca e P. L. Kale participaram da concepção do estudo, análise e interpretação dos dados, redação do artigo e aprovação da versão final. G. H. M. C. Teixeira e V. G. S. Lopes colaboraram na análise e interpretação dos dados, redação do artigo e aprovação final da versão a ser publicada.

\section{Referências}

1. VieiraMS, Vieira FM, Fröde TS, d'Orsi E. Fetal deaths in Brazil: historical series descriptive analysis 1996-2012. Matern Child Health J 2016; 20:1634-50.

2. Barros PS, Aquino EC, Souza MR. Mortalidade fetal e os desafios para a atenção à saúde da mulher no Brasil. Rev Saúde Pública 2019; 53:12.

3. Organização Mundial da Saúde. Classificação estatística internacional de doenças e problemas relacionados à saúde. 10a Revisão. São Paulo: Centro Colaborador da OMS para a Classificação de Doenças em Português; 2000.

4. Pingray V, Althabe F, Vazquez P, Correa M, Pajuelo M, Belizán JM. Stillbirth rates in 20 countries of Latin America: an ecological study. BJOG 2018; 125:1263-70.

5. Leisher SH, Teoh Z, Reinebrant H, Allanson E, Blencowe H, Erwich JJ, et al. Seeking order amidst chaos: a systematic review of classification systems for causes of stillbirth, 2009-2014. BMC Pregnancy Childbirth 2016; 16:295.

6. Wigglesworth JS. Monitoring perinatal mortality: a pathophysiological approach. Lancet 1980; 27:684-6.

7. Barbeiro FMS, Fonseca SC, Tauffer MG, Ferreira MSS, Silva FP, Ventura PM, et al. Óbitos fetais no Brasil: revisão sistemática. Rev Saúde Pública 2015; 49:22.

8. Hoffmann CBPC, Schultz LF, Vaichulonis CG, Nascimento IB, João CG, Borges DC, et al. Perinatal mortality: epidemiological profile, causes and avoidable factors at a reference public maternity hospital in the State of Santa Catarina, Brazil, 2011-2015. J Perinat Med 2020; 48:162-7.

9. Malta DC, Duarte EC. Causas de mortes evitáveis por ações efetivas dos serviços de saúde: uma revisão da literatura. Ciênc Saúde Colet 2007; 12:765-76.

10. Malta DC, Sardinha LMV, Moura L, Lansky S, Leal MC, Szwarcwald CL, et al. Atualização da lista de causas de mortes evitáveis por intervenções do Sistema Único de Saúde do Brasil. Epidemiol Serv Saúde 2010; 19:173-6.

\section{Informações adicionais}

ORCID: Sandra Costa Fonseca (0000-0001-5493494X); Pauline Lorena Kale (0000-0001-54399158); Graça Helena Maia do Canto Teixeira (00000001-6483-8695); Vania Gloria Silami Lopes (00000002-6656-5763).

11. Assis HM, Siviero PCL, Drumond EF, Machado CJ. Óbitos fetais sob o prisma da evitabilidade: análise preliminar de um estudo para o Município de Belo Horizonte. Cad Saúde Colet (Rio J.) 2014; 22:314-7.

12. Pereira RC, Figueroa MN, Barreto IC, Cabral LNC, Lemos MLC, Marques VLLR. Perfil epidemiológico sobre mortalidade perinatal e evitabilidade. Rev Enferm UFPE 2016; 10:176372.

13. Rêgo MGS, Vilela MBR, Oliveira CM, Bonfim CV. Óbitos perinatais evitáveis por intervenções do Sistema Único de Saúde do Brasil. Rev Gaúcha Enferm 2018; 39:e2017-0084.

14. Leisher SH, Teoh Z, Reinebrant H, Allanson E, Blencowe H, Erwich JJ, et al. Classification systems for causes of stillbirth and neonatal death, 2009-2014: an assessment of alignment with characteristics for an effective global system. BMC Pregnancy Childbirth 2016; 16:269.

15. Departamento de Vigilância Epidemiológica, Secretaria de Vigilância em Saúde, Ministério da Saúde. Avaliação da qualidade dos dados do óbito fetal no Sistema de Informação sobre Mortalidade do Brasil. In: Departamento de Vigilância Epidemiológica, Secretaria de Vigilância em Saúde, Ministério da Saúde, organizador. Saúde Brasil 2018 uma análise da situação de saúde e das doenças e agravos crônicos: desafios e perspectivas. Brasília: Editora do Ministério da Saúde; 2019. p. 359-75.

16. Maia LTS, Souza WV, Mendes ACG. A contribuição do linkage entre o SIM e o SINASC para a melhoria das informações da mortalidade infantil em cinco cidades brasileiras. Rev Bras Saúde Mater Infant 2015; 15:57-66.

17. Wigglesworth JS, Singer DB. Textbook of fetal and perinatal pathology. 2nd Ed. New Jersey: Wiley-Blackwell; 1998.

18. Saleem S, Tikmani SS, McClure EM, Moore JL, Azam SI, Dhaded SM, et al. Trends and determinants of stillbirth in developing countries: results from the Global Network's PopulationBased Birth Registry. Reprod Health 2018;15 Suppl 1:100. 
19. Vieira FMSB, Kale PL, Fonseca SC. Aplicabilidade da Lista Brasileira de Causas de Mortes Evitáveis por intervenção do Sistema Único de Saúde, para análise de óbitos perinatais em municípios dos estados do Rio de Janeiro e de São Paulo, 2011. Epidemiol Serv Saúde 2020; 29:e201942.

20. Aminu M, Unkels R, Mdegela M, Utz B, Adaji $\mathrm{S}$, van den Broek N. Causes of and factors associated with stillbirth in low- and middle-income countries: a systematic literature review. BJOG 2014; 121 Suppl 4:141-53.

21. Malta DC, Prado RRD, Saltarelli RMF, Monteiro RA, Souza MFM, Almeida MF. Preventable deaths in childhood, according to actions of the Unified Health System, Brazil. Rev Bras Epidemiol 2019; 22:e190014.

22. Teixeira JAM, Araujo WRM, Maranhão AGK, Cortez-Escalante JJ, Rezende LFM, Matijasevich A. Mortality on the first day of life: trends, causes of death and avoidability in eight Brazilian Federative Units, between 2010 and 2015. Epidemiol Serv Saúde 2019; 28:e2018132.

23. Saltarelli RMF, Prado RRD, Monteiro RA, Malta DC. Tendência da mortalidade por causas evitáveis na infância: contribuições para a avaliação de desempenho dos serviços públicos de saúde da Região Sudeste do Brasil. Rev Bras Epidemiol 2019; 22:e190020.

24. Lima SS, Braga MC, Vanderlei LCM, Luna CF, Frias PG. Avaliação do impacto de programas de assistência pré-natal, parto e ao recém-nascido nas mortes neonatais evitáveis em Pernambuco, Brasil: estudo de adequação. Cad Saúde Pública 2020; 36:e00039719.

25. World Health Organization. The WHO application of ICD-10 to deaths during the perinatal period: ICD-PM. Geneva: World Health Organization; 2016.

26. Martins MCF, Feitosa FEL, Viana Júnior AB, Correia LL, Ibiapina FLP, Pacagnella RC, et al. Pregnancies with an outcome of fetal death present higher risk of delays in obstetric care: a case-control study. PLoS One 2019; 14:e0216037.

27. Fonseca RMM, Garcia CL, Angimahtz TS, Battaglia CF, Chalem E, Sass N. Trends associated with stillbirth in a maternity hospital school in the north zone of São Paulo: a crosssectional study. Rev Bras Ginecol Obstet 2019; 41:597-606.

28. França GV, Restrepo-Méndez MC, Maia MF, Victora CG, Barros AJ. Coverage and equity in reproductive and maternal health interventions in Brazil: impressive progress following the implementation of the Unified Health System. Int J Equity Health 2016; 15:149.
29. Leal MDC, Szwarcwald CL, Bonilha PV, Aquino EML, Barreto M, Barros F, et al. Saúde reprodutiva, materna, neonatal e infantil nos 30 anos do Sistema Único de Saúde (SUS). Ciênc Saúde Colet 2018; 23:1915-28.

30. Cunha AC, Lacerda JT, Alcauza MTR, Natal S. Avaliação da atenção ao pré-natal na atenção básica no Brasil. Rev Bras Saúde Mater Infant 2019; 19:447-58.

31. Mario DN, Rigo L, Boclin KLS, Malvestio LMM, Anziliero D, Horta BL, et al. Qualidade do pré-natal no Brasil: Pesquisa Nacional de Saúde 2013. Ciênc Saúde Colet 2019; 24:122332.

32. Domingues RM, Leal MC, Hartz ZM, Dias MA, Vettore MV. Access to and utilization of prenatal care services in the Unified Health System of the city of Rio de Janeiro, Brazil. Rev Bras Epidemiol 2013; 16:953-65.

33. Andrews K, Bourroul MLM, Fink G, Grisi S, Scoleze Ferrer AP, Diniz EMA, et al. Time to change focus? Transitioning from higher neonatal to higher stillbirth mortality in São Paulo State, Brazil. PLoS One 2017; 12:e0190060.

34. Szwarcwald CL, Leal MDC, Esteves-Pereira AP, Almeida WDS, Frias PG, Damacena GN, et al. Avaliação das informações do Sistema de Informações sobre Nascidos Vivos (SINASC), Brasil. Cad Saúde Pública 2019; 35:e00214918.

35. Lansky S. Mortalidade fetal: mortes invisíveis e evitáveis. In: Bittencourt S, Dias M, Duarte M. organizadores. Vigilância do óbito materno, infantil e fetal e atuação em Comitês de Mortalidade. v. 1. Rio de Janeiro: Editora Fiocruz; 2013. p. 123-33.

36. Luk HM, Allanson E, Ming WK, Leung WC. Improving diagnostic classification of stillbirths and neonatal deaths using ICD-PM (International Classification of Diseases for Perinatal Mortality) codes: validation study. JMIR Med Inform 2020; 8:e20071.

37. Ruoff AB, Andrade SR, Schmitt MD. Atividades desenvolvidas pelos comitês de prevenção do óbito infantil e fetal: revisão integrativa. Rev Gaúcha Enferm 2017; 38:e67342.

38. Vanderlei LCM, Frias PG. A vigilância do óbito como instrumento para reduzir a invisibilidade da exclusão social e assistencial de mulheres e crianças. Rev Bras Saúde Mater Infant 2017; 17:635-6.

39. Frias PG, Viola RC, Navarro LM, Machado MRM, Rocha PMM, Wakimoto MD, et al. Vigilância do óbito: uma ação para melhorar os indicadores de mortalidade e a qualidade da atenção à saúde da mulher e da criança. In: Bittencourt S, Dias M, Duarte M. organizadores. Vigilância do óbito materno, infantil e fetal e atuação em Comitês de Mortalidade. v. 1. Rio de Janeiro: Editora Fiocruz; 2013. p. 201-46. 
Abstract

Although the Brazilian List of Avoidable Causes of Deaths (LBE in Portuguese), in its version for children under 5 years of age $(L B E<5)$, does not include stillbirths, some studies have used the list with or without adaptations. We present a proposal for adaptation of the LBE for stillbirths (LBE-OF in Portuguese) and the results of its application to stillbirths in the State of Rio de Janeiro, Brazil, in 2018 , compared to the results with $L B E<5$. We reviewed the categories from the 10th revision of the International Classification of Diseases (ICD-10) in the $L B E<5$ and reassigned them in the avoidability groups, according to time of death in relation to delivery and the causes consistent with stillbirths. Conditions that did not elucidate the determinants of death were allocated as ill-defined causes. Stillbirths in the State of Rio de Janeiro, selected from the databases of the Mortality Information System (SIM in Portuguese), were classified according to $L B E-O F$ and $L B E<5$. When classifying the 2,585 stillbirths that occurred in the State of Rio de Janeiro in 2018, we found that according to $L B E<5$, there were predominantly causes "reducible by adequate care in labor and delivery" (42.9\%), while according to LBE-OF, the most frequent causes were "reducible by adequate care for during pregnancy" (43.6\%). Ill-defined causes ranked second according to the LBE-OF (35.4\%) and third according to $L B E<5$. Some $30 \%$ of stillbirths changed groups and subgroups of avoidability, showing greater consistency with the profile of obstetric care. Although identifying a higher percentage of ill-defined causes, the LBE-OF is more consistent with the pathophysiology of fetal deaths. The inclusion of stillbirths in the SIM would be a positive step in monitoring and upgrading the investigation of causes of fetal death.

Fetal Death; Cause of Death; International Classification of Diseases

\section{Resumo}

A pesar de que la Lista Brasileña de Causas de Muerte Evitables (LBE), en su versión para niños menores de 5 años $(L B E<5)$, no contemple los óbitos fetales, algunos estudios la utilizaron sin o con pocas adaptaciones. Presentamos una propuesta de adaptación de la LBE para óbitos fetales (LBE-OF) y los resultados de su aplicación a óbitos fetales en el Estado de Rio de Janeiro, Brasil, en 2018, comparados con los de la $L B E<5$. Revisamos las categorías de la 10a revisión de la Clasificación Internacional de Enferemedades (CIE-10) presentes en la $L B E<5, y$ las asignamos en los grupos de evitabilidad, según el momento del óbito, en relación con el parto y las causas acordes con óbitos fetales. Las condiciones que no aclararon los determinantes del óbito fueron asignadas como causas mal definidas. Los óbitos fetales del Estado de Río de Janeiro -seleccionados de las bases del Sistema de Información sobre Mortalidad (SIM)- se clasificaron según el LBE-OF y la LBE < 5. Al clasificar los 2.585 óbitos fetales del Estado de Rio de Janeiro ocurridos en 2018, se observó que, según la $L B E<5$ años, predominarían causas "reducibles por una adecuada atención al parto" (42,9\%), mientras que por la LBE-OF, aquellas "reducibles por una adecuada atención a la mujer en la gestación" fueron las más frecuentes (43,6\%). Las causas mal definidas ocuparon la segunda posición según la $L B E-O F$ (35,4\%), y la tercera posición según $L B E<5$. Cerca de un $30 \%$ de los óbitos fetales cambiaron de grupos y subgrupos de evitabilidad, mostrando una mayor coherencia con el perfil de atención obstétrica. A pesar de haberse identificado un mayor porcentaje de causas mal definidas, la LBE-OF es más compatible con la fisiopatología de los óbitos fetales. Su inserción en el SIM avanzaría en el sentido de monitorear y calificar la investigación de causas de muerte fetal.

Muerte Fetal; Causas de Muerte; Classificación Internacional de Enfermedades
Recebido em 09/Set/2020

Versão final reapresentada em 01/Dez/2020

Aprovado em 08/Dez/2020 